\title{
Ultra-High Energy Cosmic Rays, entering a new Era of Astroparticle Physics and Detection Techniques
}

\section{Olaf Scholten*, for the Pierre Auger Collaboration}

KVI, University of Groningen, Groningen, The Netherlands

E-mail: scholten@kvi.nl

Recent results obtained for ultra-high energy cosmic-ray detection with the Pierre Auger Observatory are presented. The importance of a hybrid detector is stressed. In addition, plans are presented for extending this capability with the detection of radio signals that are emitted by cosmic-ray initiated air-shower in the atmosphere of the Earth.

From Planets to Dark Energy: the Modern Radio Universe

October 1-5 2007

The University of Manchester, $U K$

${ }^{*}$ Speaker. 


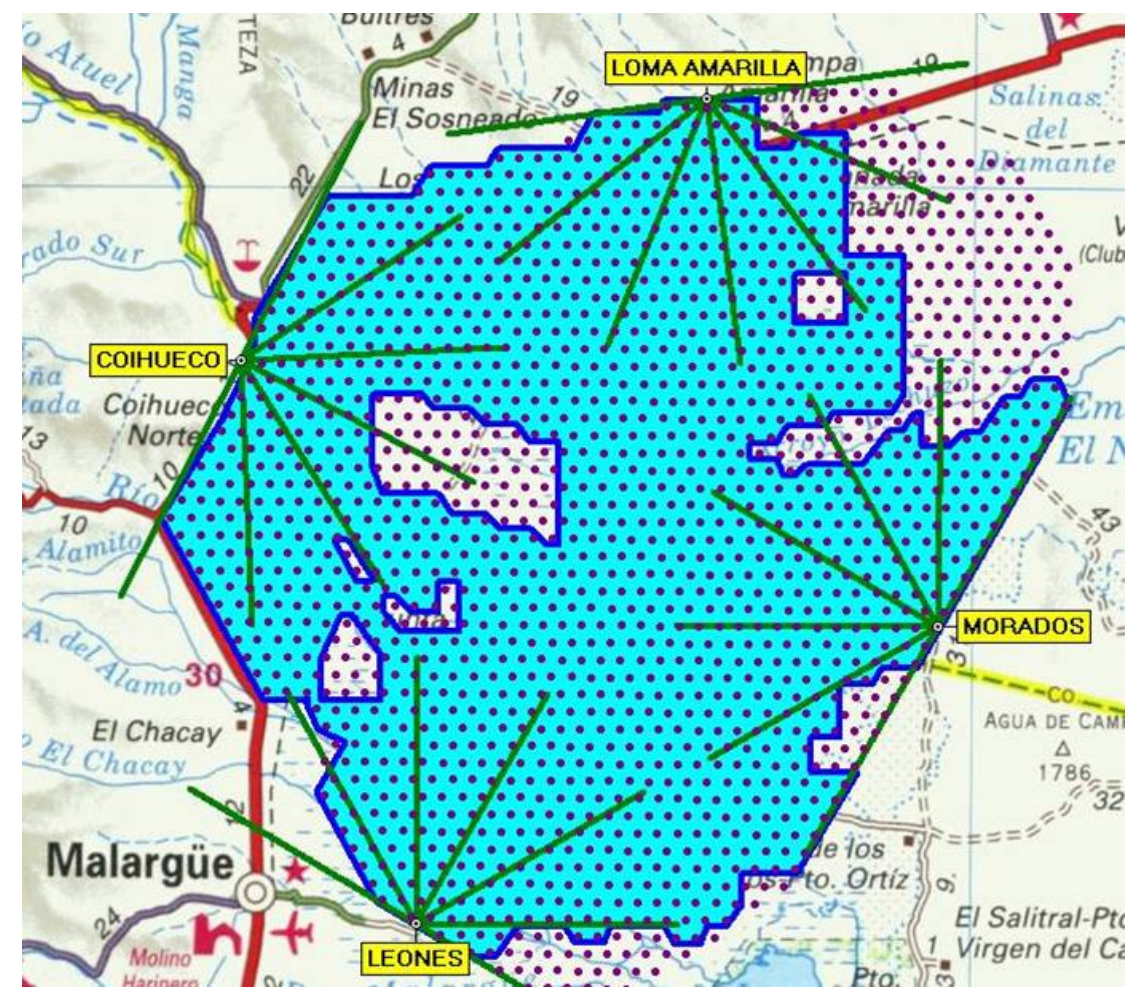

Figure 1: Layout of the southern site of the Pierre Auger Observatory. The dots represent the position of each of the 1600 SD stations. The shaded area indicates the SD stations that are deployed and activated as of the summer of 2007. The green lines indicate the field of view of the six telescopes at each FD site.

\section{The Pierre Auger Observatory}

The Pierre Auger Observatory is situated near the town of Malargüe in Argentina where an area of $3000 \mathrm{~km}^{2}$ is being covered with water-Cherenkov detectors with a separation distance of $1.5 \mathrm{~km}$ on a triangular grid. This grid is now for more than $90 \%$ in place and operational and should be completed early 2008. In addition to these Surface Detectors (SD) there is also a system of Fluorescence Detectors (FD) situated in four buildings overlooking the site as shown in Fig. 1. The individual water tanks are indicated by the dots in the figure where the shaded area indicates the detectors that are placed and are in operation. The lines fanning out from four different points indicate the lines of sight of the FD cameras in the four FD buildings.

An Ultra-High Energy (UHE) particle entering the atmosphere will induce there an Extensive Air Shower (EAS), a large cascade of particles. Some of these particles, mainly electrons and muons, will reach the Earth and can be detected in the surface detectors. The size of this footprint is directly related to the energy of the initial cosmic ray and its arrival direction. The latter can be determined from the differences in the arrival time of the particles in the different surface detectors.

In the EAS air molecules are ionized and emit fluorescence light on recombination. The amount of emitted light is proportional to the energy deposited by the particles in the shower which allows for an accurate tracing of the shower profile, the number of charged particles as function of height in the atmosphere. In addition this measurement gives an independent determination of the 


\begin{tabular}{c|ccc} 
& hybrid & SD only & FD mono \\
\hline Angular resolution & $\sim 0.3^{\circ}$ & $\sim 1^{\circ}-2^{\circ}$ & $\sim 3^{\circ}-5^{\circ}$ \\
Aperture & E free & E free & E dependent \\
Energy & M free & M dependent & M free
\end{tabular}

Table 1: The advantage [3] of hybrid detection is indicated. The label "E (M) free" implies that, for a particular detection scheme, the aperture or energy determination for cosmic rays is -to a large extent- independent of their energy (E) (model (M)) used in the simulation.

arrival direction of the cosmic ray.

The southern site of the Pierre Auger Observatory needs to cover an area of $3000 \mathrm{~km}^{2}$ in order to have a large aperture to detect UHE cosmic rays. Due to this large collecting area, combined with the hybrid detection capability, the facility is the leading facility for cosmic-ray research at the highest energies.

The hybrid detection scheme used at the Pierre Auger observatory allows the detection of air showers by two very different systems, SD and FD. The combination of the recorded information of both components makes it possible to reconstruct the energy, the arrival direction, the composition, and the flux of cosmic rays with a precision that is vastly superior to what can be reached with only a single system [1,2]. From Table 1, adapted from Ref. [3], it can be seen that the angular resolution is strongly improved for hybrid events. In addition, the determination of the energy of the cosmic ray and the total aperture for detecting cosmic ray can be done model independently. Model dependence enters mostly through the assumptions for the first few collisions in the shower where the energies are well beyond what can experimentally be accessed on Earth with accelerators like the ones at CERN. With only SD measurements the reconstructed energy depends rather strongly on Monte Carlo simulations.

The disadvantage of the present hybrid detection scheme is that the FD system can operate only on moon-less, cloudless nights and thus has an up time of the order of $10 \%$. The surface detectors do not have such limitations. This greatly hampers the efficiency for hybrid event detection. For the physics, as discussed in the following section, a good energy determination and angular resolution is imperative. To enhance the hybrid detection capability we are developing radiometric detection of the electro-magnetic pulse emitted by the EAS.

\section{Recent Results}

Recently the Pierre Auger Observatory has considerably improved the data sets on three fronts that are of interest in UHE cosmic-ray physics, namely, the isotopic composition of the cosmic rays, the energy spectrum, and the correlation between arrival directions and nearby extra-galactic source candidates.

The present data [4] indicate that even at the highest energies, where there is sufficient statistics, the composition of the cosmic rays is a mixture between protons and heavier nuclei, usually taken as iron. The analysis of the relative abundances is based on the elongation rate of the air shower. For this the hybrid detection capability of Pierre Auger is essential. The analysis is however model dependent since it relies on a detailed comparison with EAS simulations. The search 


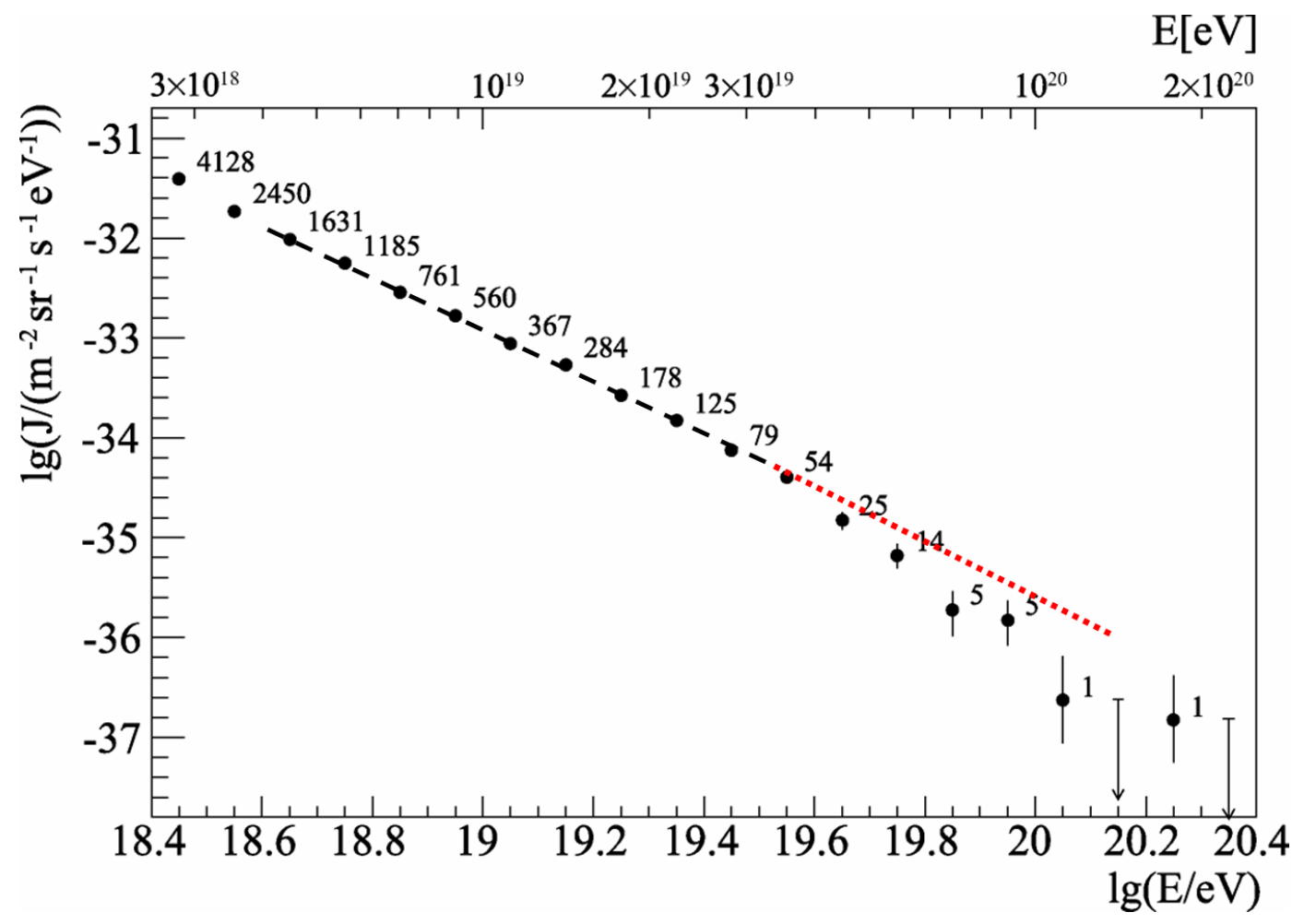

Figure 2: The energy spectrum [7] of the cosmic rays as determined by the Pierre Auger Observatory.

for events induced by UHE gamma-rays does not suffer from this model dependence and strong limits [5] have been set.

The important issue of the cosmic-ray energy spectrum at the highest energies is to study the presence of the so-called GZK mechanism which would lead to reduction in the flux spectrum at energies higher than $60 \mathrm{EeV}\left(1 \mathrm{EeV}=10^{18} \mathrm{eV}\right)$. The physics of the GZK mechanism is that at the highest energies the protons are sufficiently energetic to produce pions when scattering off the cosmic microwave background. The prediction relies on the validity of Lorentz invariance even for particles with a Lorentz factor of $\gamma=10^{11}$. Lorentz invariance could be broken by the existence of extra dimensions which are rolled-up at a scale that corresponds to these high energies. In the recent data of the Pierre Auger Observatory there is clear evidence for a strong extra reduction in the flux [6, 7] at an energy of $60 \mathrm{EeV}$, see Fig. 2, as expected on the basis of the GZK mechanism. Based on a power-law extrapolation of the spectrum one expects to find at energies in excess of $40 \mathrm{EeV}, 132 \pm 9$ events while only 51 are detected, similarly, at energies in excess of $100 \mathrm{EeV}$, $30 \pm 3$ events are predicted while seeing only 2 , which is a very significant (six sigma) depletion of the flux.

The most interesting recent observation, published in Science magazine [8], is that at energies above $55 \mathrm{EeV}$ cosmic rays appear to originate from points in the sky, which correlate strongly with the positions of Active Galactic Nuclei. In Fig. 3 the arrival directions of UHE cosmic rays are indicated by small circles and the positions of AGN within a distance of $75 \mathrm{Mpc}$ by stars. This correlation is a very significant observation for several reasons. The fact that the sources for UHE cosmic rays are not randomly distributed over the sky excludes some of the more exotic 


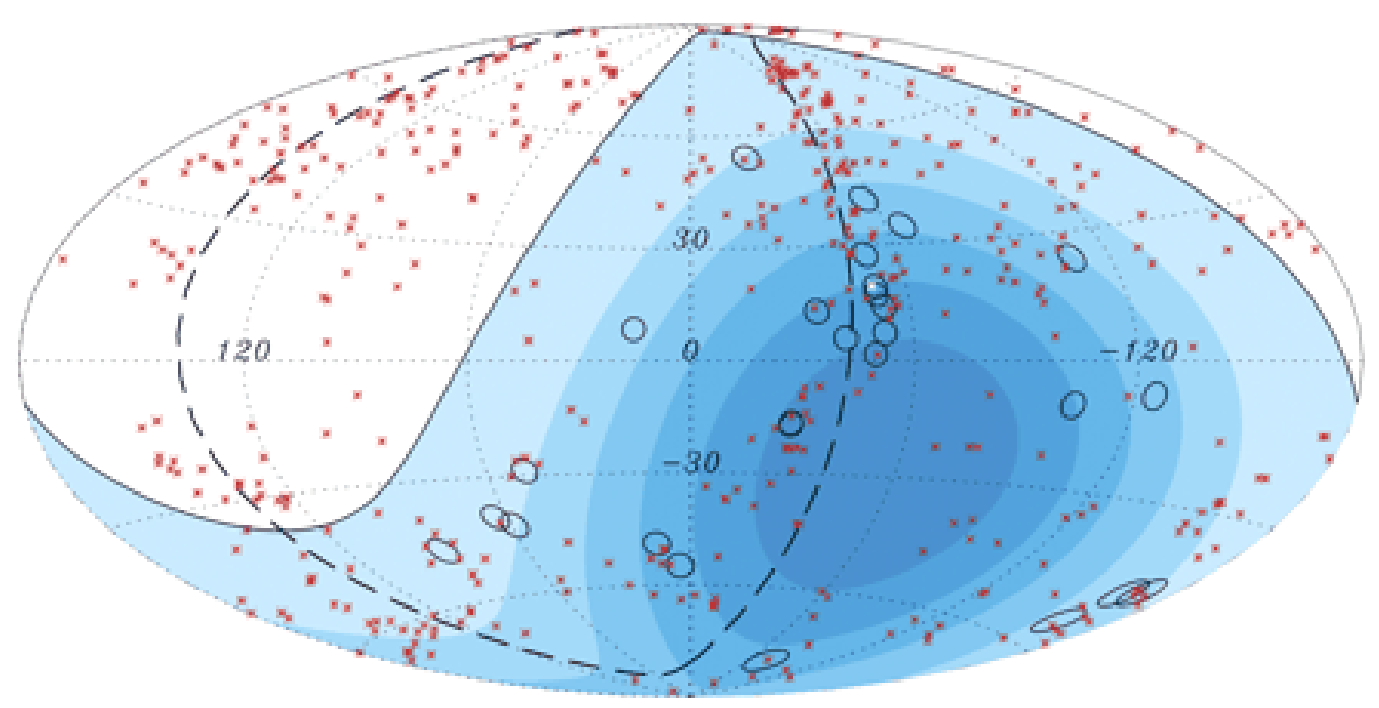

Figure 3: The arrival directions of cosmic rays with energies above $60 \mathrm{EeV}$ show a strong correlation with the positions of nearby AGN [8].

possibilities for their origin such as homogeneously distributed topological defects formed at the time of the big-bang. Instead it indicates that they come from astronomical objects which opens the possibility of particle astronomy. The sources for these UHE-cosmic rays might indeed lie in the AGN for which the correlations are observed or some other astronomical objects that correlate strongly in position with the AGNs. Whatever it is, it must be very spectacular objects that are able to accelerate particles to these enormous energies.

Another important aspect of recently observed correlations is the confirmation of the GZK effect. The sources appear to be within the attenuation distance of about $75 \mathrm{Mpc}$ for cosmic rays of energies in excess of $55 \mathrm{EeV}$. For lower energies the universe will become transparent. The lower-energy cosmic rays will be able to reach Earth for all sources anywhere in the universe. As a consequence one thus would expect to observe a rather uniform distribution of sources at lower energies, which is consistent with observation.

In addition the fact that the UHE-cosmic rays keep their pointing accuracy over distances of $75 \mathrm{Mpc}$ implies that the magnetic deflection of these charged particles over these distances is less that the correlation precision of $3^{\circ}$.

With the prospect of performing particle astronomy it is important to do a full sky map, necessitating the opening of a site in the northern hemisphere of the Earth since with the southern observatory only part of the sky is covered. To determine the actual sources and to be able to map the intergalactic magnetic field from the energy dependence of the deflections it is necessary to improve the statistics by one or two orders of magnitude. A high pointing accuracy is required, necessitating the hybrid detection capability. Hybrid detection is also necessary for determining the isotopic composition of the cosmic rays through their shower profile. Since the fluorescence detectors can operate for only a small fraction of the time (moonless, cloudless nights) it will be very interesting to explore other more efficient methods. One possibility is offered by the detection of the radio pulse by the air shower as discussed in the following section. 


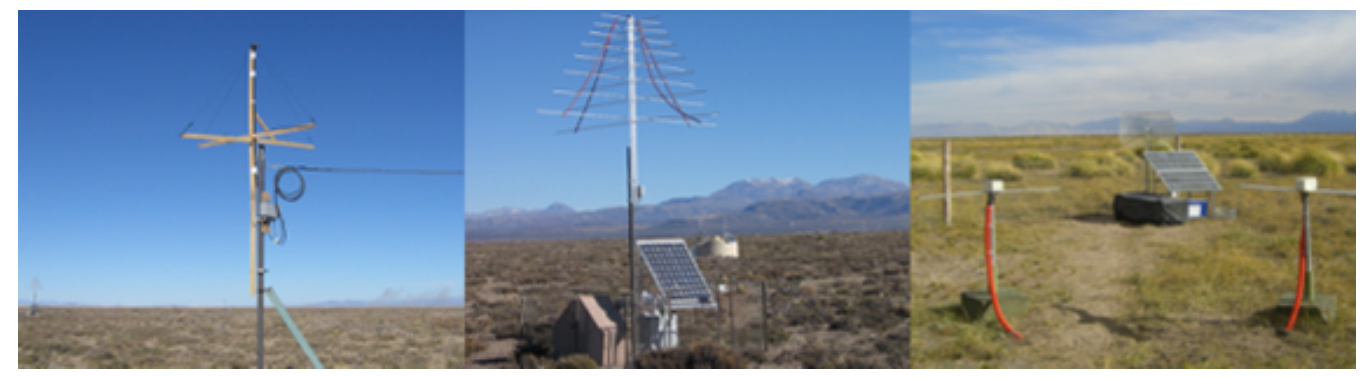

Figure 4: Antenna's that have been deployed at the Pierre Auger site, from left to right, a dipole of the type used for LOFAR, a log-periodic dipole (LPDA), and a dipole of the type used in the CODALEMA array.

\section{Radio detection at the Pierre Auger Observatory}

In recent years there has been a strong revival in the interest in the use of radio detection for cosmic-ray induced air showers with the promising results obtained from recent LOPES $[9,10]$ and CODALEMA $[11,12]$ experiments. These experiments have in turn triggered plans to install an array of radio detectors at the Pierre Auger Observatory [13, 14]. First, I will discuss our present understanding of the radio-emission mechanism from EAS and then the plans for deployment of radio antennas at the Pierre Auger Observatory.

Already in the earliest publications on radio emission from air showers [15, 16, 17, 18], the importance of coherent emission was stressed. Two mechanisms, Cherenkov radiation and geomagnetic radiation were proposed as possibilities, where the latter mechanism indicates that the interaction of the charged particles in the EAS with the Earth magnetic field drives the emission of radio waves. In more recent work [19], the picture of coherent synchrotron radiation from secondary shower electrons and positrons gyrating in the Earth's magnetic field was proposed. Very recently [20], a calculation was presented in the coherent geo-magnetic picture, indicating a simple algebaï relation between the time development of the pulse and the longitudinal shower profile. In this picture it is argued that the acceleration of the electrons induced by the Lorentz force is compensated by a deceleration due to collisions with air molecules resulting in a relatively constant average drift velocity in a direction which is perpendicular to the shower and the magnetic field.

Some first experimental antenna systems have been deployed at the Pierre Auger Observatory. In Fig. 4 a few of the different antenna's are shown. All antennas are sensitive in the frequency range of $25-70 \mathrm{MHz}$ where there are relatively few broadcasting stations. A typical power spectrum [13] is shown in Fig. 5. It is shown in Ref. [14], that the power in the spectrum around $50 \mathrm{MHz}$ shows a clear variation with a period of the length of a sidereal day, and not local time. This provides clear indication that the dominant background component is galactic noise and that the instrumental noise is well under control.

A typical signal as registered by the experimental antenna system is shown in Fig. 6. It is initiated by a $2 \mathrm{EeV}$ cosmic ray entering the atmosphere at a zenith angle of about $60^{\circ}$ with the impact point at a distance of $300 \mathrm{~m}$ from the antennas. The signals in the different antennas are clearly polarized in a direction perpendicular to the direction of the magnetic field as predicted by the models, see Fig. 6. 


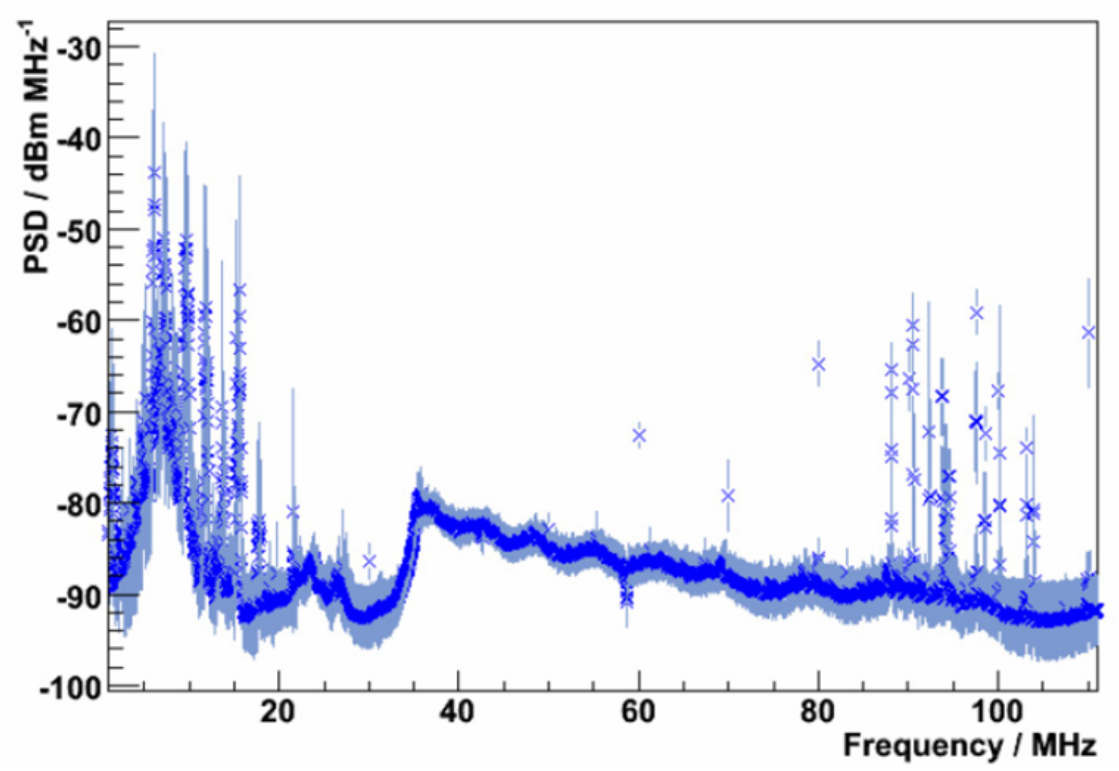

Figure 5: A typical spectral power density measured [21] with an LPDA at the Pierre Auger observatory.
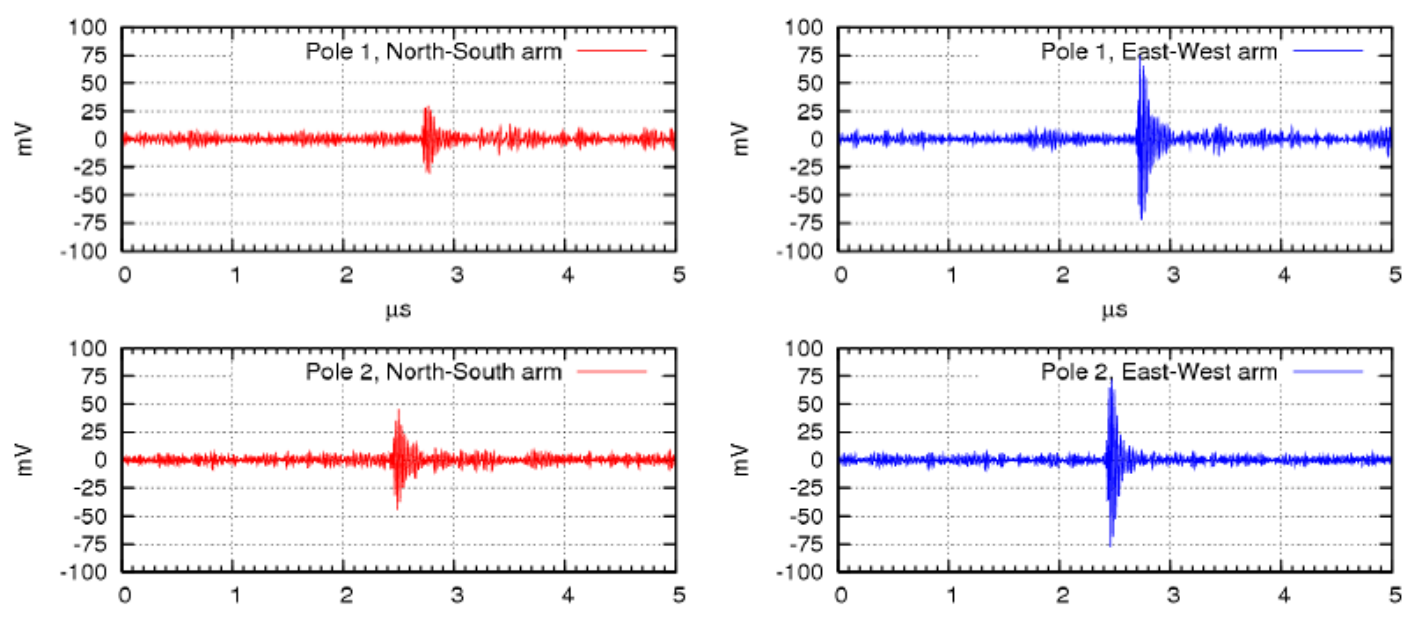

$\mu \mathrm{s}$
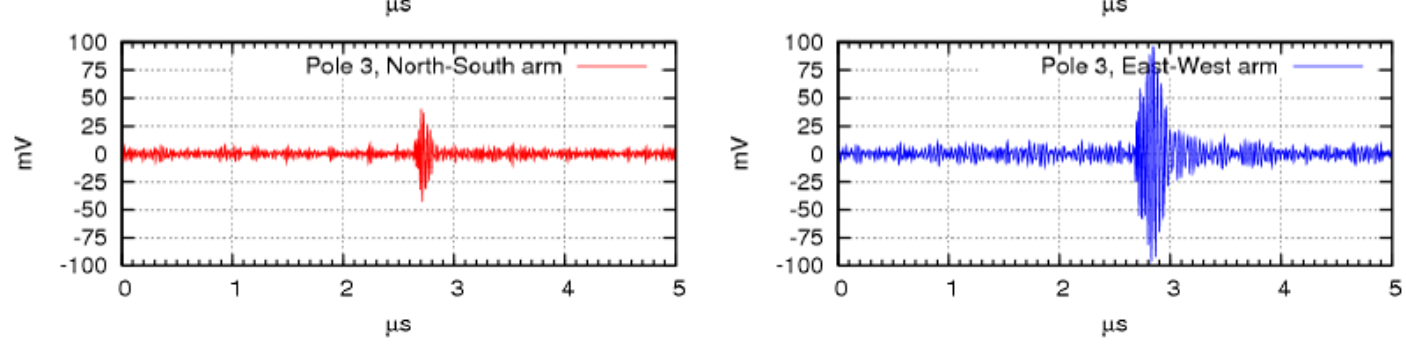

Figure 6: Typical signals picked up by the radio antennas. 
The next stage in the development of radio antennas is to deploy an engineering array covering an area of $20 \mathrm{~km}^{2}$ at the southern site of the Pierre Auger Observatory. Before this can be done several technical issues have to be resolved. In order to handle the enormous data stream produced by the digital antennas a sophisticated trigger scheme has to be developed. Since a large area is to be covered a wireless system for the data transfer from the antennas to the central collecting point should be installed. Furthermore, each station has to have its own solar-power system which also implies that all electronics at the antenna station should be low in power consumption. All electronics should be designed such that the RFI noise produced is well below the galactic noise level. Tests concerning these challenges for a future system have already started since the end of 2006, at two small test sites at the Pierre Auger Observatory. Considerable progress has been made, however, a particularly difficult problem to solve is to build autonomous antennas with a low power consumption $(<10 \mathrm{~W})$.

\section{Conclusions}

In this presentation I have shown that we are at the brink of an exciting new era with radiometric detection of cosmic rays. At the Pierre Auger Observatory it promises to allow for hybrid detection of cosmic rays at the highest energies with full efficiency. There is evidence that the information extracted from radiometric detection is complementary to that obtained from the surface and the fluorescence detectors. At energies that are even beyond the capability of the Pierre Auger Observatory a promising option is offered by the NuMoon project [22], the radiometric detection of cosmic ray and neutrino impacts on the moon using the LOFAR and SKA telescope arrays.

\section{References}

[1] M. Mostafa for the P. Auger Collaboration, Proc. Cosmic Ray Int. Sem., CRIS 2006, Catania, Italy, Nucl. Phys. B (Proc. Suppl.) 165 (2007) 50-58.

[2] B.R. Dawson for the P. Auger Collaboration, Proc. of the $30^{\text {th }}$ Int. Cosmic-Ray Conf. (ICRC), Merida, Mexico, 2007; Astro-ph 0706.1105.

[3] A. Watson, Highlight talk at the $30^{\text {th }}$ Int. Cosmic-Ray Conf., Merida, Mexico, 2007.

[4] Michael Unger et al., Proc. of the $30^{\text {th }}$ Int. Cosmic-Ray Conf. (ICRC), Merida, Mexico, 2007, \#594.

[5] J. Abraham et al. [Pierre Auger Collaboration], Astroparticle Physics 27 (2007), 155; (astro-ph/0606619).

[6] Markus Roth et al., Proc. of the $30^{\text {th }}$ ICRC, Merida, Mexico, 2007, \#313.

[7] Tokonatsu Yamamoto et al., Proc. of the $30^{\text {th }}$ ICRC, Merida, Mexico, 2007, \#318.

[8] The Pierre Auger Collaboration, Science, vol.318, p.938-943.

[9] H. Falcke, et al., Nature 435, 313 (2005).

[10] W. D. Apel, et al., Astropart. Physics 26, 332 (2006).

[11] D. Ardouin et al., Nuclear Instr. Method (NIM) A 555, 148 (2005).

[12] D. Ardouin, et al., Astropart. Physics 26, 341 (2006). 
[13] Ad van den Berg et al., Proc. of the $30^{\text {th }}$ ICRC, Merida, Mexico, 2007, \#176.

[14] C. Timmermans for the P. Auger collaboration, Subm. to the $10^{\text {th }}$ Int. Conf. on Advanced Technology and Particle Physics, October 8-12, 2007, Como, Italy (2007).

[15] J. V. Jelley et al., Nature 205, 327 (1965).

[16] N.A. Porter, et al., Phys. Lett. 19, 415 (1965).

[17] F.D. Kahn and I.Lerche, Proc. Royal Soc. London A289, 206 (1966).

[18] H. R. Allan, Prog. in Element. part. and Cos. Ray Phys. 10, 171 (1971).

[19] T. Huege, H. Falcke, Astronomy \& Astrophysics 412, 19 (2003).

[20] O. Scholten, K. Werner, and F. Rusydi, To appear in Astroparticle Physics; Proc. of the $30^{\text {th }}$ ICRC, Merida, Mexico, 2007, \#69

[21] Tobias Winchen, Diploma thesis, 2007, University of Aachen.

http://web.physik.rwth-aachen.de/ hebbeker/theses/winchen_diploma.pdf

[22] O. Scholten, et al., Astropart. Phys. 26 (2006) 219. 